\title{
Analysis on the Growth and Characterization of a Non-linear Optical Single Crystal: L-Cystine Dihydrobromide
}

\author{
Azeezaa Varsha Mohammed ${ }^{a, b}$, Joseph Arul Pragasam Arulappan ${ }^{c}$, Sunitha Thangaiya Ganesan ${ }^{d}$ \\ Suresh Sagadevan ${ }^{e *}$ \\ ${ }^{a}$ Research Scholar, Department of Physics, Sathyabama University, Chennai- 600 119, India \\ ${ }^{b}$ Department of Physics, SRR Engineering College, Chennai- 603 103, India \\ ${ }^{c}$ Department of Physics, Sathyabama University, Chennai- 600 119, India \\ ${ }^{d D e p a r t m e n t ~ o f ~ C h e m i s t r y, ~ P a c h a i y a p p a ' s ~ C o l l e g e, ~ C h e n n a i-~} 600$ 030, India \\ ${ }^{e}$ Department of Physics, AMET University, Kanathur, Chennai - 603112, India
}

Received: June 15, 2015; Revised: July 3, 2015

\begin{abstract}
Nonlinear optical single crystals of L-cystine dihydrobromide (LCHB) have been grown by slow evaporation method. Single crystal X-ray diffraction analysis revealed the crystal system and lattice parameter values. Powder X-ray diffraction analyses have been carried out and the diffraction patterns have been indexed. Fourier transform infrared (FTIR) analysis confirms the various functional groups present in the grown crystal. The thermal behavior of the grown crystal was investigated by DTA and TGA analysis. The optical properties of the crystals were determined using UV-Visible transmittance spectrum. The dielectric constant and dielectric loss of the crystal are studied as a function of frequency for various temperatures. The SHG efficiency of the crystal is studied using the Kurtz and Perry technique.
\end{abstract}

Keywords: growth from solution, $x$-ray diffraction, infrared spectra, optical transmission spectrum

\section{Introduction}

L-cysteine $\left[\mathrm{C}_{3} \mathrm{H}_{7} \mathrm{NO}_{2} \mathrm{~S}\right]$ is the simplest amino acid and it has centre of chirality and is optically active. L-cysteine can exist as neutral molecule in the gas phase; it exists as a Zwitterion in solution as well as in solid state. L-cysteine crystallizes in non-centrosymmetric space group making it a potential candidate for piezoelectric and non-linear applications. L-cysteine exist as a dipolar ion in solid state in which carboxyl group is present as a carboxylate ion and amino group is present as ammonium ion. In addition, thiol group is present in aqueous solution of L-cysteine. Due to this dipolar nature, L-cysteine has a high melting point. Another added advantage of L-cysteine is the presence of chromophores namely amino group and carboxyl group which makes it transparent in the UV-Vis region ${ }^{1,2}$. Thus more emphasis has been given by the scientists to develop non-linear optical crystals in L-cysteine and its analogs. Experiments conducted by Martin et al. ${ }^{3}$, Loganayaki et al. ${ }^{4}$, Bhagavannarayana et al. ${ }^{5}$, Selvaraju et al. ${ }^{6}$ and Anbuchezhiyan et al. ${ }^{7}$ reveal the suitability of L-cysteine family crystals for their non-linear optical properties and applications. The non-linear optical properties of L-cysteine analogs make them strong candidates to replace KDP for frequency conversion of infrared lasers. All compounds of this class contain optically active carbon atoms and therefore all of them form acentric crystals ${ }^{8}$. The present study deals with L-cystine dihydrobromide (LCHB), one of the non-linear optical crystals belonging to L-cysteine family. The growth of the crystal has been achieved by slow solvent evaporation technique. The grown crystals were characterized by single crystal XRD analysis, powder XRD, FTIR, thermal analysis, DSC, UV analysis, dielectric and SHG studies.

\section{Experimental Procedure}

\subsection{Crystal growth}

L-cystine dihydrobromide $\left(\mathrm{C}_{6} \mathrm{H}_{14} \mathrm{Br}_{2} \mathrm{~N}_{2} \mathrm{O}_{4} \mathrm{~S}_{2}\right)$ crystals are grown from aqueous solution by slow solvent evaporation technique. The starting materials are L-cysteine and hydrobromic acid. The solution is prepared by dissolving equimolar amount of 2:2 of L-cysteine and hydrobromic acid in double distilled water and the solution is stirred using a magnetic stirrer having hot plate attachment. The mixed solution was slowly heated up to $60^{\circ} \mathrm{C}$ and a saturated solution was obtained. This solution is filtered using micro filter paper of $10 \mu \mathrm{m}$. The filtered saturated solution is transferred into a petri dish. The prepared solution is allowed to evaporate at room temperature and kept in undisturbed condition. Large size single crystals were obtained due to the collection of monomers at the seed crystal sites from the mother solution, after the nucleation and growth processes were completed. LCHB crystal of dimension about $10 \times 10 \times 6 \mathrm{~mm}^{3}$ was harvested in a growth period of twenty four days by slow evaporation of the solvent. The photograph of the grown LCHB crystal is shown in Figure 1. During the growth of LCHB, the formation of several funguses like organism in the solution is observed. These organisms start growing on the surface, exposed to atmosphere and subsequently sink into the solution, thereby, contaminate it. Due to this reason,

*e-mail: sureshsagadevan@gmail.com 
bigger size crystals could not be grown. A similar problem is also reported during the growth of $\mathrm{LAP}^{9}$ and $\mathrm{GSN}^{10}$. Therefore attempt is made to inhibit the growth of these microbes by using $\mathrm{H}_{2} \mathrm{O}_{2}$. A good quality crystal is obtained by repeated recrystallization.

\subsection{Solubility}

The size of the crystal depends on the amount of the material available in the solution which decides the solubility of the material in that solvent for solution growth technique ${ }^{11}$. The solubility of synthesized material in water is performed by maintaining the water at constant temperature and adding the known quantity of the material till it dissolves completely. Then the solution is stirred continuously for 8hrs and a small quantity of the solution is pipetted out and its decomposition is determined gravimetrically. The variation of solubility with temperature of LCHB is shown in Figure 2. LCHB has a positive temperature coefficient of the solubility. Hence slow cooling as well as slow evaporation techniques can be employed for the growth of bigger sized crystals.

\section{Results and Discussion}

\subsection{Single crystal $X$-ray diffraction}

The grown crystals are subjected to single crystal X-ray diffraction studies using MESSRS ENRAF NONIUS, the Netherlands diffractometer. It is observed that LCHB crystal belongs to orthorhombic system with a non-centrosymmetric space group of $\mathrm{P} 22_{1} 2_{1}$. The cell parameters are $\mathrm{a}=7.42 \AA$, $\mathrm{b}=17.69 \AA, \mathrm{c}=5.35 \AA, \alpha=\beta=\gamma=90^{\circ}$ and $\mathrm{V}=707.68 \AA^{3}$, which agree well with the available reported literature values ${ }^{[7]}$.

\subsection{Powder XRD analysis}

The powdered sample of LCHB is subjected to powder $\mathrm{X}$-ray diffraction studies. The X-ray diffraction analysis for LCHB is performed with a high resolution automatic X-ray diffractometer. Finely ground powder of the crystals was subjected to intense X-rays of wavelength $\lambda=1.5406 \AA$ with a scan speed of $0.001^{\circ}$ per min. Intensities for the diffraction peaks were recorded over the range of $(2 \theta)$ $20-80^{\circ}$ and indexed using computer programs. Figure 3 represents the powder X-ray diffractogram for the grown LCHB. The lattice parameters are evaluated as: $\mathrm{a}=7.48 \AA$, $\mathrm{b}=17.85 \AA, \mathrm{c}=5.35 \AA$ and $\alpha=\beta=\gamma=90^{\circ}$ and the results are found to be in good agreement with the results of single crystal XRD.

\subsection{FTIR analysis}

In order to qualitatively analyze the presence of functional groups in LCHB, Fourier Transform Infrared (FTIR) spectrum is recorded in the range 4000-500 $\mathrm{cm}^{-1}$ using the BRUKER IFS $66 \mathrm{~V}$ infrared spectrophotometer. The characteristic absorption peaks are shown in Figure 4. L-cysteine exists in zwitterions in which the absorption due to carboxyl group is observed at 892.8 and $1614 \mathrm{~cm}^{-1}$, respectively. In LCHB, these peaks are shifted to 866 and $1625 \mathrm{~cm}^{-1}$, respectively. Similarly, the absorption peaks due to amino $\left(\mathrm{NH}_{3}^{+}\right)$group of L-cystine are observed at 1111, 1195 and $1631 \mathrm{~cm}^{-1}$, respectively. These are shifted to 1134 , 1187 and $1625 \mathrm{~cm}^{-1}$, respectively. The sharp and strong band at $1734 \mathrm{~cm}^{-1}$ indicates the $\mathrm{C}=\mathrm{O}$ stretching of $\mathrm{COOH}$ group. Nevertheless, from the presence of carboxylate ion and ammonium ion it may be concluded that L-cystine molecule exist in zwitterionic form in LCHB. The S-S stretching vibrations of LCHB are observed at $745 \mathrm{~cm}^{-1}$. The S-S vibration shows the presence of disulphide bond in LCHB crystal. The absorption peaks due to thiol S-H group of L-cysteine lies in 2546 or $2540 \mathrm{~cm}^{-1}$ and this S-H stretching mode is shifted to 2521 or $2562 \mathrm{~cm}^{-1}$ in case of LCHB. Additional evidences for the zwitterionic monolayer

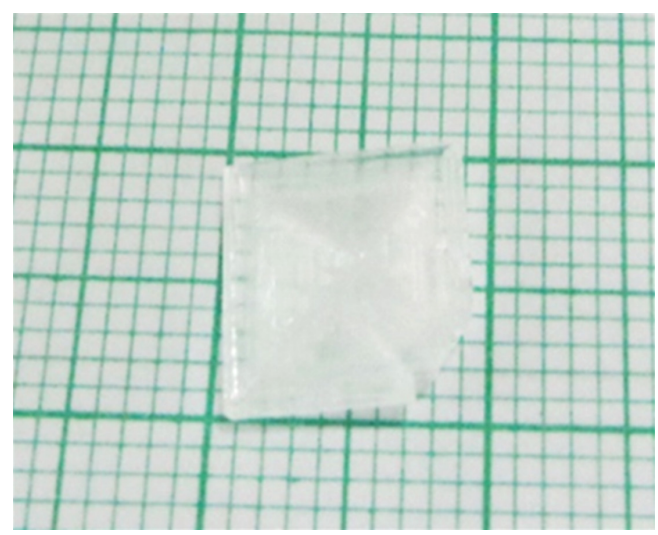

Figure 1. Photograph of as-grown LCHB crystal.

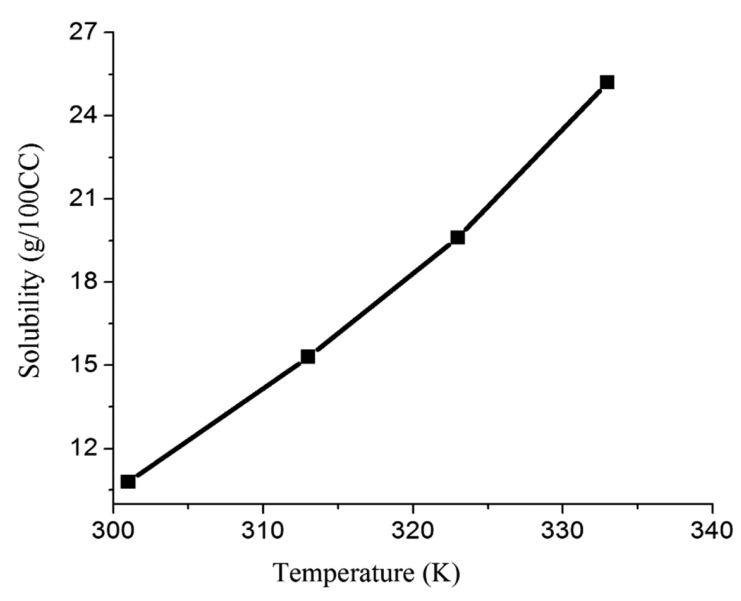

Figure 2. Solubility curve of LCHB crystal.

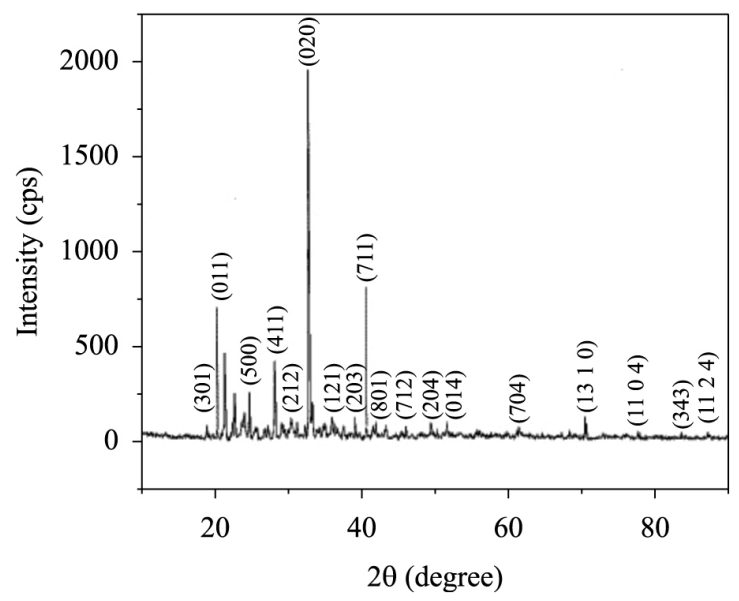

Figure 3. Powder XRD pattern of LCHB crystal. 
is the in-plane bending and asymmetric stretching assigned to the $1215-866 \mathrm{~cm}^{-1}$ region ${ }^{12,13}$. The sulfur containing amino acid cystine vibrations, in particular S-S and S-H stretching vibrations, are comparable with pure and complexes of L-cystine. The protonation of cystine with hydrogen bromide is also confirmed by the above discussion.

\subsection{UV-Vis transmittance spectrum}

The UV-Visible transmittance spectrum was recorded using a Varian Cary 5E model UV-Vis-NIR spectrometers in the range $200-1400 \mathrm{~nm}$ are displayed in Figure 5. It is observed that the crystal is transparent in the entire visible region and the absorption takes place in the UV range between $259-320 \mathrm{~nm}$. The maximum absorption takes place at a wavelength of $265 \mathrm{~nm}$. This maximum absorbance at $265 \mathrm{~nm}$ is assigned to $\pi-\pi^{*}$ electron transition and may be attributed to the excitation in the resonance hybrid of $\mathrm{C}=\mathrm{O}$ group. This is a further indication of the presence of unionized $\mathrm{COOH}$ group of the cystine in the grown LCHB crystal. The absence of absorption in the visible region is the intrinsic property of all amino acids and its substituted crystals. The absorption with in UV range is again the property of amino acids and its substituted crystals. This property is the key requirement for the NLO materials and other related optoelectronic applications ${ }^{14}$.

\subsection{TG-DTA analysis}

The thermogravimetry (TGA) and Differential Thermal Analysis (DTA) are employed to identify the phase transition, different stages of decomposition and melting point of the grown

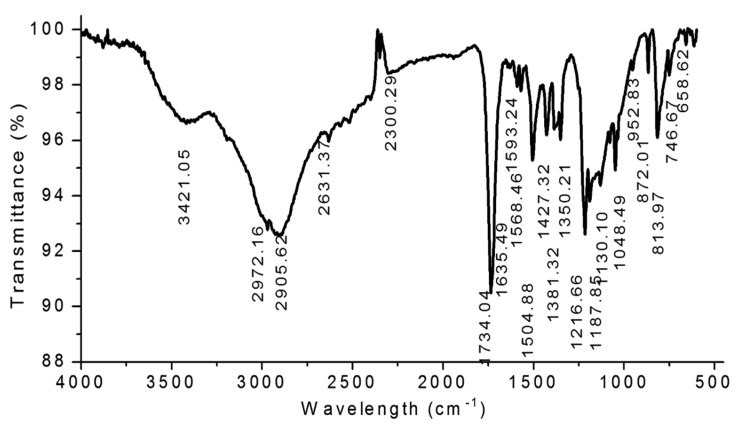

Figure 4. FTIR spectrum of LCHB crystal.

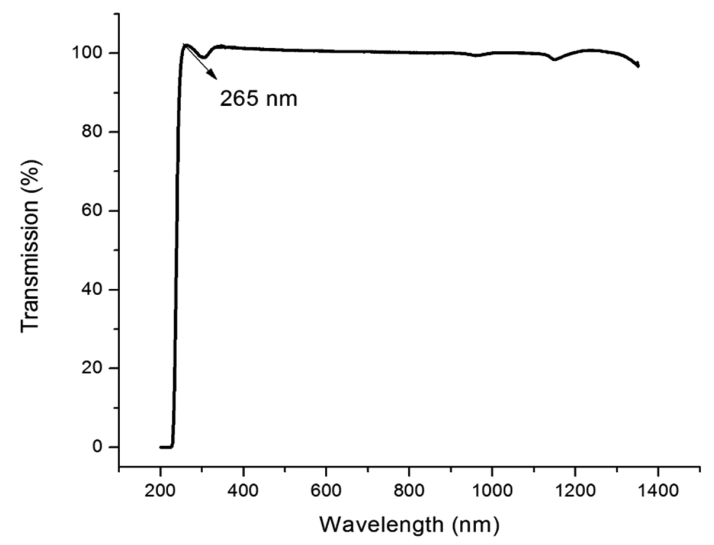

Figure 5. UV-Vis-NIR spectrum of LCHB crystal. crystal $^{15}$. The TGA and DTA analysis of LCHB are carried out between $20^{\circ} \mathrm{C}$ and $1000^{\circ} \mathrm{C}$ in the nitrogen atmosphere with a heating rate of $10^{\circ} \mathrm{C} / \mathrm{min}$ using a SDTQ600 V8 analyzer. The TGA and DTA curve is shown in Figure 6. In TGA there is a small peak at $65^{\circ} \mathrm{C}$ which may be due to loss of water molecule. There is a sharp exothermic peak with a maximum at $203^{\circ} \mathrm{C}$ which corresponds to the major weight loss of about $28.36 \%$. Hence this exothermic peak is assigned to melting point of the compound. The sharpness of this exothermic peak shows the good degree of crystallinity of the grown crystal. The subsequent exothermic peaks that follow this melting correspond to the decomposition of the compound in four stages. First stage occurs in the region of $231-254^{\circ} \mathrm{C}$. Weight loss observed in this region is about $16 \%$ and this is assigned to loss of $\mathrm{NH}_{3}$ and carbon dioxide molecules. Second stage of decomposition occurs in the region $465-538^{\circ} \mathrm{C}$, the residue loses its $11 \%$ of weight which corresponds to loss of thiol group. Third stage of decomposition starts at $745^{\circ} \mathrm{C}$, in this stage further decomposition of residue occurs. The DTA analysis of LCHB confirms that there is an endothermic peak below $200^{\circ} \mathrm{C}$ which is due to loss of water. Thus isomorphic transitions within the crystals are ruled out below its decomposition temperature. Hence LCHB crystal is expected to have more lattice force in the crystal lattice.

\subsection{DSC analysis}

The DSC analysis of LCHB powder of the grown crystal is carried out between $20^{\circ} \mathrm{C}$ and $1000^{\circ} \mathrm{C}$ in the nitrogen atmosphere with a heating rate of $10^{\circ} \mathrm{C} / \mathrm{min}$ using a SDTQ600 V8 analyzer. The resulting spectrum is shown in Figure 7. An endothermic peak is observed at $75.94^{\circ} \mathrm{C}$, which is assigned to the loss of weakly absorbed water. It is also coinciding with the weight loss in the DTA trace. As the crystal is hygroscopic this may be due to the adsorbed water of the grown crystals. The intense endotherm above $194.89^{\circ} \mathrm{C}$ also agrees with the first intense weight loss in the TGA trace, it is assigned to be the decomposition temperature. The melting point of the material is determined to be $200^{\circ} \mathrm{C}$. The decomposition process continues up to $500^{\circ} \mathrm{C}$ with the removal of almost all the compound as gaseous products. Hence, from this study, it can be said that the crystal can retain texture up to $200^{\circ} \mathrm{C}$. However, the compound undergoes isomorphic transformation at $200^{\circ} \mathrm{C}$; its application is restricted up to $200^{\circ} \mathrm{C}$ only.

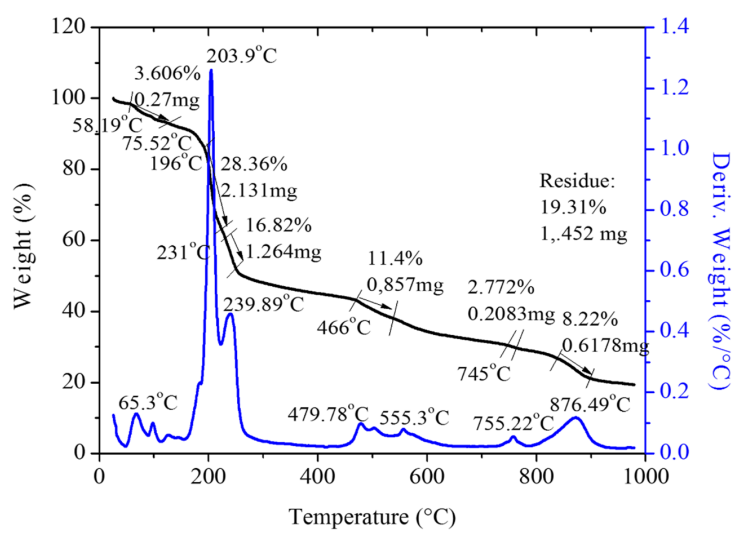

Figure 6. TG-DTA curve of LCHB crystal. 


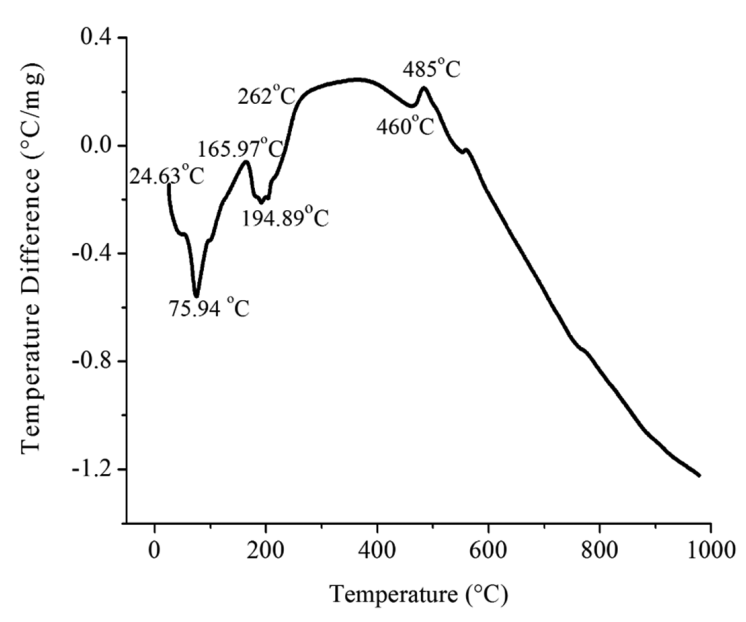

Figure 7. DSC curve of LCHB crystal.

\subsection{Dielectric studies}

The dielectric study on LCHB single crystal is carried out using HIOKI 3532 LCR HITESTER. A sample of dimension $3.27 \times 2.78 \times 0.98 \mathrm{~mm}^{3}$ having silver coating on opposite faces is placed between the two copper electrodes and thus a parallel plate capacitor is formed. The capacitance of the sample was measured by varying the frequency from $50 \mathrm{~Hz}$ to $5 \mathrm{MHz}$ for various temperatures $(313 \mathrm{~K}, 333 \mathrm{~K}, 353 \mathrm{~K}$ and $373 \mathrm{~K})$. Figure 8 shows the plot of dielectric constant versus frequency for various temperatures. The very high value of dielectric constant at low frequency may be attributed to space charge polarization. At lower range of frequencies dielectric constant decreases drastically with frequency, becoming a constant at larger frequencies. The polarization occurs due to the local displacement of electrons, which is the effect of the electronic exchange of the number of ions in the crystal. As frequency increases the electron exchange cannot follow the electric field and polarization becomes less dependent of frequency. Variation of dielectric constant with temperature is generally attributed to the orientation polarization, crystal expansion, the presence of impurities and crystal defects. When temperature increases the dielectric constant decreases, as evident from the Figure 8. The thermal energy disrupts the ion dipole interaction which is responsible for polarization at higher temperatures, causing the relaxation of polarization. Also the dielectric loss is plotted against frequency and is shown in Figure 9. These curves suggest that the dielectric loss is strongly dependent on the frequency of the applied field, similar to that of dielectric constant. This behavior is common in the case of ionic systems ${ }^{16,17}$.

\subsection{SHG studies}

The second harmonic generation efficiency is evaluated by Kurtz and Perry powder technique ${ }^{18}$ and microcrystalline powder of KDP is taken as reference material. The crystal of LCHB is illuminated using Q-switched, mode locked Nd:YAG laser of wavelength $\lambda=1064 \mathrm{~nm}$ of pulse width $10 \mathrm{~ns}$ and the corresponding second harmonic output power has been recorded. The output from the crystal was monochromated to collect the intensity of $532 \mathrm{~nm}$ component and to eliminate the fundamental wavelength. The second harmonic radiation generated by the randomly oriented micro crystals was

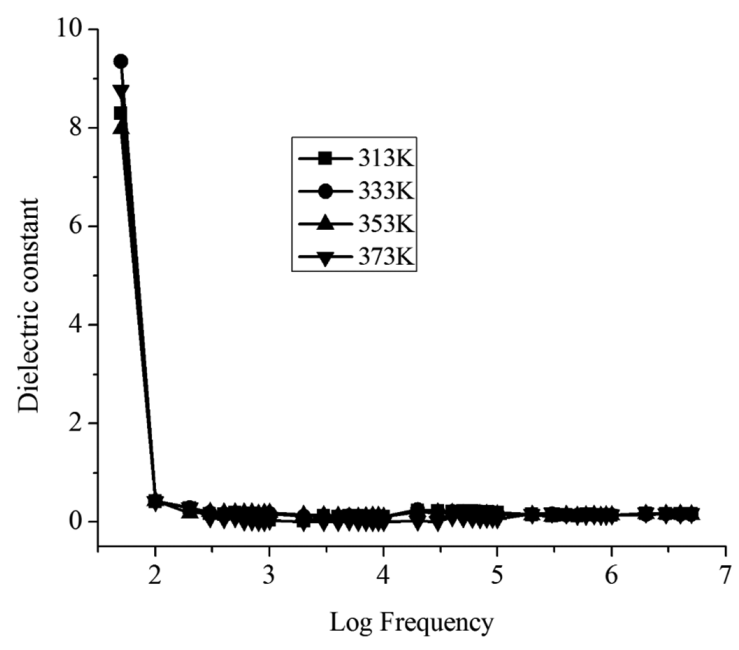

Figure 8. Dielectric constant versus log frequency of LCHB crystal.

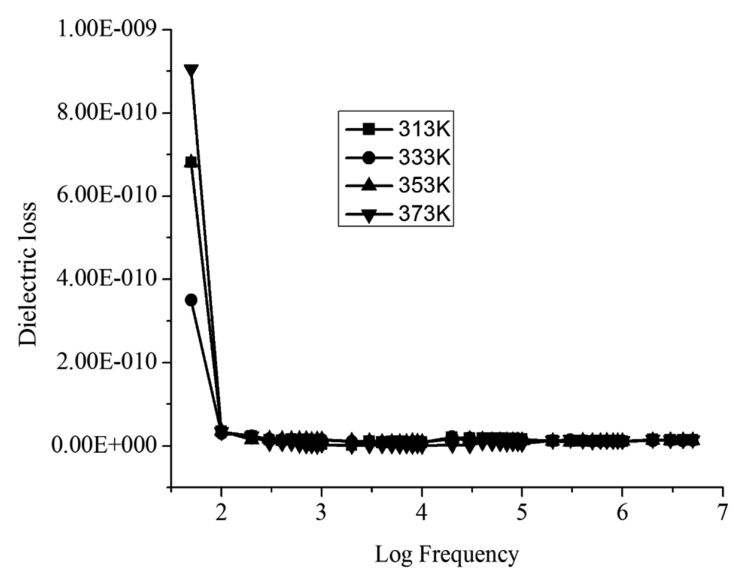

Figure 9. Dielectric loss versus log frequency of LCHB crystal.

focused by a lens and detected and a photo multiplier tube. The generation of the second harmonic was confirmed by a strong bright green emission emerging from the powdered crystal. A potassium dihydrogen phosphate (KDP) crystal was used as a reference material in the SHG measurement. The relative conversion efficiency was calculated from the output power of LCHB crystals with reference to KDP crystals. It is observed that the conversion efficiency of LCHB is 0.42 times that of KDP crystal.

\section{Conclusion}

Bulk sized LCHB single crystals were grown by slow evaporation technique. Single crystal XRD and powder XRD confirmed the lattice parameters. The functional groups were confirmed from FTIR analysis. The optical property of the grown material is studied by UV-Vis spectroscopy which shows the UV cut off wavelength to be $265 \mathrm{~nm}$. The TG-DTA and DSC analysis depicted that the crystal is thermally stable up to $200^{\circ} \mathrm{C}$. The dielectric constant and dielectric loss measurements are carried out at different temperatures and frequencies. The NLO property of the LCHB crystal was examined by performing Kurtz powder test using Nd: YAG laser. 


\section{References}

1. Tlahuice-Flores A. Zwitterion L-cysteine adsorbed on the Au20 cluster: enhancement of infrared active normal modes. Journal of Molecular Modeling. 2013; 19(4): 1937-1942.

2. Tarakeshwar P and Manogaran S. Vibrational frequencies of cysteine and serine zwitterions -an- ab- Initio assignment. Spectrochimica Acta Part A: Molecular and Biomolecular Spectroscopy. 1995; 51(5):925-928. http://dx.doi.org/10.1016/05848539(94)00233-2.

3. Martin Britto Dhas SA and Natarajan S. Growth and characterization of two new NLO materials from the amino acid family: L-Histidine nitrate and L-Cysteine tartrate monohydrate. Optics Communications. 2008; 281(3):457-462. http://dx.doi. org/10.1016/j.optcom.2007.09.049.

4. Loganayaki M and Murugakoothan P. Growth, structural and dielectric behavior of L-Cysteine hydrochloride monohydrate single crystals. Asian Journal of Chemistry. 2011; 23:50855088 .

5. Bhagavannarayana G, Kumar S, Shakir M, Kushawaha SK, Maurya KK, Malhotra R, et al. Unidirectional growth of L-cysteine hydrochloride monohydrate: first time observation as nonlinear optical material and its characterization. Journal of Applied Crystallography. 2010; 43(4):710-715. http://dx.doi. org/10.1107/S0021889810016870.

6. Selvaraju K, Valluvan R, Kirubavathi K and Kumararaman S. L-Cystine hydrochloride: a novel semi-organic nonlinear optical material for optical devices. Optics Communications. 2007; 269(1):230-234. http://dx.doi.org/10.1016/j.optcom.2006.07.075.

7. Anbuchezhiyan M, Ponnusamy S and Muthamizhchelvan C. Crystal growth and characterizations of L-cystinedihydrobromide - a semiorganic nonlinear optical material. Physica B, Condensed Matter. 2010; 405(4):1119-1124. http://dx.doi.org/10.1016/j. physb.2009.10.061.

8. Monaco SB, Davis LE, Velsko SP, Wang FT, Eimerl D and Zalkin A. Synthesis and characterization of chemical analogs of 1-arginine phosphate. Journal of Crystal Growth. 1987; 85(12):252-255. http://dx.doi.org/10.1016/0022-0248(87)90231-4.
9. Yokotani A, Sasaki T, Fujioka K, Nakai S and Yamanaka C. Growth and characterization of deuterated L-arginine phosphate monohydrate, a new nonlinear crystal, for efficient harmonic generation of fusion experiment lasers. Journal of Crystal Growth. 1990; 99(1-4):815-819. http://dx.doi.org/10.1016/ S0022-0248(08)80032-2.

10. Bhat MN and Dharmaprakash SM. New nonlinear optical material: glycine sodium nitrate. Journal of Crystal Growth. 2002; 235(1-4):511-516. http://dx.doi.org/10.1016/S00220248(01)01799-7.

11. Christian R. Solvents and solvent effects in organic chemistry. New York: VCH; 1990.

12. Pavia DL, Lampman GM and Kritz GS. Introduction to spectroscopy. 3rd ed. Fort Worth: Harcourt College Publishers; 2001.

13. Pawlukojc A, Padureanu I, Aranghel D. L-Cysteine: Neutron spectroscopy, Raman, IR and ab initio study. Spectrochimica Acta Part A Molecular and Biomolecular Spectroscopy. 2005; 61(11-12):2474-2481.

14. Vijayan N, Ramesh Babu R, Gopalakrishnan R, Dhanuskodi S and Ramasamy P. Growth of semicarbazone of benzophenone single crystals. Journal of Crystal Growth. 2002; 236(1-3):407417. http://dx.doi.org/10.1016/S0022-0248(01)02207-2.

15. Gonsago CA, Albert HM, Umamaheswari R and Pragasam AJA. Spectral, optical and thermal studies of pure and $\mathrm{Zn}$ (II)-doped L-histidine hydrochloride monohydrate (LHHC) crystals. Journal of Thermal Analysis and Calorimetry. 2012; 110(2):839-845. http://dx.doi.org/10.1007/s10973-011-1850-9.

16. Sagadevan S. Studies on optical, mechanical, dielectric properties of bisthiourea nickel bromide NLO single crystal. Optik International Journal for Light and Electron Optics. 2014; 125(22):6746-6750. http://dx.doi.org/10.1016/j.ijleo.2014.08.059.

17. Suresh S and Arivuoli D. Growth, theoretical, optical and dielectric properties of 1 tartaric acid NLO single crystals. Journal of Optoelectronics and Biomedical Materials.2011; 3:63-68.

18. Kurtz SK and Perry TT. A powder technique for the evaluation of non-linear optical materials. Journal of Applied Physics. 1968; 39(8):3798-3813. http://dx.doi.org/10.1063/1.1656857. 